\title{
Effects of organochlorine pesticides (OCPs) on survival and edibility of loaches in the World Heritage Honghe Hani Rice Terraces, China
}

\author{
Chao Song ${ }^{1,2,3,4, *}$, Jingwei Zhang ${ }^{1,2,3,4, *}$, Gengdong $\mathrm{Hu}^{2,3,4}$, Yi Yin ${ }^{5,6}$, Liping $\mathrm{Qiu}^{2,3,4}$, \\ Limin Fan ${ }^{2,3,4}$, Yao Zheng ${ }^{2,3,4}$, Shunlong Meng ${ }^{2,3,4}$, Cong Zhang ${ }^{2,3,4}$, Jiazhang Chen ${ }^{1,2,3,4, * *}$ \\ ${ }^{1}$ Wuxi Fisheries College, Nanjing Agricultural University, 214081 Wuxi, PR China \\ ${ }^{2}$ Freshwater Fisheries Research Center, Chinese Academy of Fishery Sciences, 214081 Wuxi, PR China \\ ${ }^{3}$ Laboratory of Quality \& Safety Risk Assessment for Aquatic Products on Environmental Factors (Wuxi), \\ Ministry of Agriculture and Rural Affairs, 214081 Wuxi, PR China \\ ${ }^{4}$ Key Laboratory of Control of Quality and Safety for Aquatic Products, Ministry of Agriculture and Rural Affairs, \\ 100000 Beijing, PR China \\ ${ }^{5}$ Pearl River Fisheries Research Institute, Chinese Academy of Fishery Sciences, 510380 Guangzhou, PR China \\ ${ }^{6}$ Ministry of Agriculture and Rural Affairs Laboratory of Quality \& Safety Risk Assessment for Aquatic Product (Guangzhou), \\ 510380 Guangzhou, PR China
}

\begin{abstract}
A new rice-loach co-culture system was introduced into the Honghe Hani Rice Terraces (HHRT), China, to promote sustainable development of this World Heritage region. However, little is known about the survival of loaches in this area and whether they are safe to eat after having been exposed to residual organochlorine pesticides (OCPs). Although some OCPs such as dichlorodiphenyl trichloroethanes (DDTs), hexachlorocyclohexanes (HCHs), aldrin, and dieldrin have been banned in China, they can still be detected in the agricultural environment. In this study, water, sediment, and loaches (water and sediment collected 3 times; loaches only at harvest) in the HHRT were simultaneously sampled throughout the culture season and analyzed for 22 selected OCPs. Results showed that the concentration of total OCPs in the water and sediment uniformly decreased in the following order May > August > October and that samples collected during August and October clustered together in nMDS analysis, differing from those collected in May. Some OCPs, such as heptachlor, heptachlor epoxide, endosulfan I, endosulfan II, DDTs and HCHs were present in abundance. DDTs and HCHs were persistently present in low sediment concentrations at $0.21-1.27$ and $0.01-0.95 \mu \mathrm{g} \mathrm{kg}^{-1}$, respectively, suggesting that a new origin of these pesticides may exist. Heptachlor accounted for the majority of OCPs at the beginning of the co-culture season and was calculated to influence the survival of loaches. Residual OCPs were generally not detected in the muscle of loaches (i.e. the edible part of loaches) being sent to market and therefore pose a low risk to humans who eat them. Overall, the results of this study will be useful in the production and consumption of loaches in rice-loach coculture systems, as well as for the promotion of sustainable development of the HHRT.
\end{abstract}

KEY WORDS: Organochlorine pesticides $\cdot$ Terraces $\cdot$ Loaches $\cdot$ Survival $\cdot$ Edibility $\cdot$ Risk assessment

\section{INTRODUCTION}

Because of the drastically increasing world population, global food security is becoming increasingly fragile (Godfray et al. 2010). Land limitations in

\footnotetext{
${ }^{*}$ These authors contributed equally to this work

${ }^{* *}$ Corresponding author: ffrcchen@hotmail.com
}

mountainous regions face particularly great challenges in terms of producing sufficient amounts of food (Xie et al. 2011). Accordingly, people have improved planting patterns to adapt to harsh environments (Mäder et al. 2002, Singh 2007). One example

(C) The authors 2019. Open Access under Creative Commons by Attribution Licence. Use, distribution and reproduction are unrestricted. Authors and original publication must be credited. 
is the Honghe Hani Rice Terraces (HHRT), which comprise a well-known World Agricultural Heritage Site in southern Yunnan Province, China (Gu et al. 2012, Zhang et al. 2017). In order to meet the needs of development, our group introduced a rice-loach coculture system in the local area. The terraces were built to store water, preserve soil, and increase production; the introduction of the rice-loach system was not in conflict with these objectives. Local residents are willing to co-culture rice and loaches Misgurnus anguillicaudatus. There is a loach nursery in the local area to facilitate the supply of loach seedlings. According to a production survey carried out by local farmers, the output of loaches increased to $750 \mathrm{~kg} \mathrm{ha}^{-1}$, and the rice yield increased from 10500 to about $12750 \mathrm{~kg} \mathrm{ha}^{-1}$ after the introductioin of the rice-loach co-culture system

Inevitably, pests, weeds, and pathogens infiltrate almost all paddy soils, including rice terraces (Kim \& Smith 2001). Organochlorine pesticides (OCPs), such as dichlorodiphenyl trichloroethanes (DDTs) and hexachlorocyclohexanes (HCHs) (Lal et al. 2010), have been widely applied as pesticides for several decades. Although most OCPs were banned between the 1970s and 1990s, they may still remain in the soil (Qiu et al. 2005, Mahmoud et al. 2016).

OCPs in paddy soils and overlying water may influence the survival of aquatic organisms newly introduced by the co-culture system. Exposure to OCPs has been reported to be associated with the disruption of antioxidant enzymes and neurotransmitters (Mrema et al. 2013) and to induce behavioral changes in aquatic organisms (Daley et al. 2014). Accordingly, the acute or chronic toxicity of different OCPs has been tested in some staple aquatic organisms (Singh 2016); however, little is known about the safe exposure concentration of OCPs with respect to loaches. Additionally, in the rice-loach co-culture system, loaches feed on natural sources which are exposed to OCPs (e.g. phytoplankton and zooplankton) rather than on formulated feeds. Thus, the risks posed by OCPs on the entire loach food chain in paddy soils should be addressed, with the purpose of assessing the safety of loaches as food for humans.

Residual OCPs can be found in the edible muscle of aquatic products being sold at market. For example, Zhang et al. (2016) measured concentrations of 21 OCP residues in loach species inhabiting rice paddies in northeast China and found that DDTs and $\mathrm{HCHs}$ accounted for over $97 \%$ of the total OCPs. Residual OCPs in loaches were high or the OCPs present were harmul, thus making the consumption of loach a dietary risk for humans (Zhang et al. 2016).
Moreover, organic chlorine has been detected not only in the rice field system but also in natural systems. In particular, DDTs have frequently been found in aquatic products that were captured in natural systems (e.g. reservoir, river, or lake) (Fu et al. 2003, Zhang et al. 2010, Zhao et al. 2013). Inferentially, as a category of persistent organic pollutants (POPs), the OCPs are threatening human health via the food web. Thus, it is essential to determine how residual OCPs remaining in the HHRT influence the survival of loaches and assess their safety as a food product in the newly introduced rice-loach co-culture system.

In the present work, water and sediment in the HHRT were collected 3 times throughout the culture season (pre-culture in May, mid-term in August, and harvest in October). Loach samples were only collected during harvest, and 22 OCPs were analyzed using a gas chromatography-tandem electron capture detector (GC-ECD). The objective of this study was to explain how OCPs influence the survival of loaches cultured in paddy soils and evaluate the human health risk associated with their consumption. Further analysis should help us establish guidlines to promote the production and consumption of loaches in the rice-loach co-culture system in the World Heritage HHRT.

\section{MATERIALS AND METHODS}

\subsection{Study area and sampling strategy}

The village of Nimei in Honghe County was selected as the study area (Fig. 1). It is located at a high altitude in the core area of the HHRT and contains a terrace with an area of approximately 35 ha. The agricultural water sources in the area are from the Red Star Reservoir, the only reservoir in the area. Water and sediment were collected from 9 evenly placed sampling locations at 3 time points throughout the culture season (May, August, and October); loaches were collected during the harvest season in October. The purpose of our study was to explore the effects of OCPs throughout the culture season. Each sampling site represented an area of farmland completely retained by a constructed edge.

Water samples were collected $10 \mathrm{~cm}$ below the surface, and sediment was collected from the top of paddy soils. Overall, 54 water and sediment samples were collected during the study. Loach samples were collected with the help of local residents and were only collected in October shortly before the time when the loaches would be sent to market. The sam- 


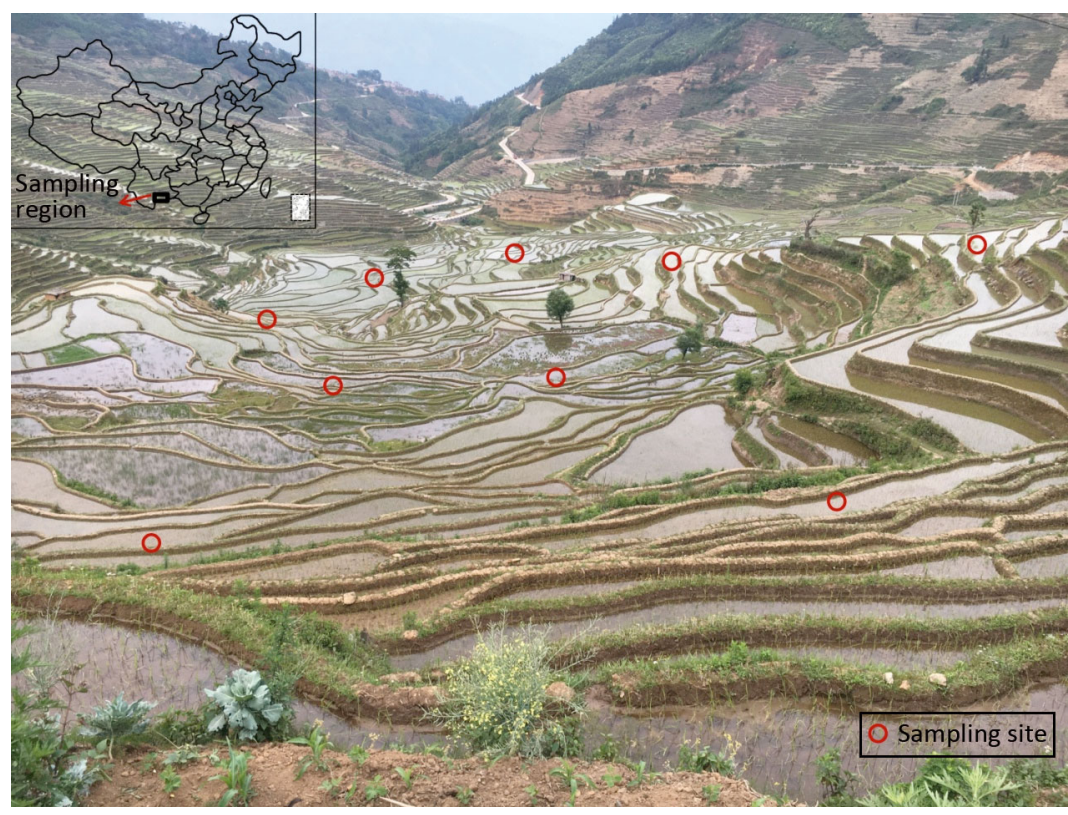

Fig. 1. Sampling sites uniformly placed in the village of Nimei, located in Honghe County in the core area of the Honghe Hani Rice Terraces

Briefly, $250 \mathrm{ml}$ water samples were transferred into a separating funnel, after which $40 \mathrm{ml}$ hexane:dichloromethane (1:1, vol:vol) was added to extract the OCPs. Subsequently, the supernatant was concentrated by rotary evaporation for subsequent instrumental analysis.

Next, approximately $5 \mathrm{~g}$ freezedried sediment was weighed and transferred into a centrifuge tube, after which $20 \mathrm{ml}$ hexane:acetone (1:1, vol:vol) was added, mixed, and centrifuged for extraction. The supernatant was then cleaned with anhydrous sodium sulfate and florisil solid phase extraction (SPE) columns. SPE proceeded as follows: $5 \mathrm{ml}$ acetone: hexane (1:9, vol:vol) was used for activation, after which the supernatant was loaded into the column and then eluted with $6 \mathrm{ml}$ hexane. The elution solutions were concen-

pling area was divided into 3 regions; 3 sampling sites were set up in each region, and 3 loach samples were collected at each sampling site and were combined to form one sample, yielding a total of 9 samples. All samples were transported to the laboratory for pretreatment immediately upon collection.

\subsection{Chemicals}

A total of 22 OCPs, including DDTs $\left(o, p^{\prime}-\mathrm{DDT}, p, p^{\prime}-\right.$ $\mathrm{DDT}, o, p^{\prime}$-DDD, $p, p^{\prime}$-DDD, $o, p^{\prime}-\mathrm{DDE}, p, p^{\prime}-\mathrm{DDE}$, and methoxychlor), HCHs ( $\alpha-\mathrm{HCH}, \beta-\mathrm{HCH}, \gamma-\mathrm{HCH}$, and $\delta$-HCH), heptachlors (heptachlor and heptachlor epoxide), drins (aldrin, isodrin, dieldrin, and endrin), endosulfans (endosulfan I and endosulfan II), chlordane, mirex, and hexachlorobenzene, were selected for analysis in the present study. All OCPs standards and internal standards $\left({ }^{13} \mathrm{C}\right.$-labeled stock solutions) were over $98 \%$ pure and purchased from the Dr. Ehrenstorfer Company. Dichloromethane, hexane, and acetone used for pretreatment were of chromatography grade and were purchased from Merck.

\subsection{Pretreatment and instrumental analysis}

Samples were pretreated and quantified as described in related research (Zhang et al. 2016) and the literature (Sun et al. 2016), with minor modifications. trated for instrumental analysis.

The edible part of the loaches, i.e. muscle, was cut into small pieces with scissors, then homogenized. Approximately $2 \mathrm{~g}$ of freeze-dried muscle tissue was then used to extract OCPs by accelerated solvent extraction (ASE; BUCHI) using procedures similar to those used for sediment.

A GC-ECD (Agilent-7890A) was used to analyze the 22 OCPs. All compounds were separated using a DB-5 capillary column $(30 \mathrm{~m} \times 0.25 \mathrm{~mm}$; i.d. $=0.25$ $\mu \mathrm{m})$. The oven temperature was programmed as follows: initial temperature $100^{\circ} \mathrm{C}$, which was held for $1 \mathrm{~min}$, followed by an increase to $250^{\circ} \mathrm{C}$ at $30^{\circ} \mathrm{C} \mathrm{min}^{-1}$ and then a further increase to $290^{\circ} \mathrm{C}$, where it was held for $5 \mathrm{~min}$. For accurate quantification and rigorous quality assurance, method detection limits (MDLs) and recoveries were calculated. The MDLs, which were defined as $10 \times$ the signal-to-noise ratio, were 2.13-4.21 $\mathrm{ng} \mathrm{l}^{-1}, 0.12-0.41 \mu \mathrm{gg}^{-1}$, and 0.51-9.50 $\mu \mathrm{g}$ $\mathrm{kg}^{-1}$ for water, sediment, and loach samples, respectively. The ranges of recovery were 45.51-110.12, $52.34-118.15$, and $50.20-115.23 \%$ for water, sediment, and loach samples, respectively.

\subsection{Data processing}

Total OCPs, total DDTs, and total HCHs were denoted as OCPs, DDTs, and HCHs, respectively; see Table 1 for ranges and mean values. To determine the 
distribution of water and sediment samples, non-metric multidimensional scaling (nMDS) was used in the R platform (Oksanen et al. 2006). Further data was analysed as described below to assess the survival risk of loaches and the risks posed to humans who consume them.

\subsection{Survival risk of HHRT loaches}

The living environment, including water and sediment, jointly influence the survival of loaches due to their benthic habits. Thus, the survival risk quotient (SRQ) was defined as the mean value of risk characterized by water and sediment, namely $S R Q_{\text {water }}$ and $\mathrm{SRQ}_{\text {sediment, }}$ respectively, that is, the SRQ was calculated using Eq. (1):

$$
\mathrm{SRQ}=\mathrm{SRQ}_{\text {water }}+\mathrm{SRQ}_{\text {sediment }} / 2
$$

Here, $S_{\text {water }}$ was calculated using Eq. (2):

$$
\mathrm{SRQ}_{\text {water }}=\mathrm{MC}_{\mathrm{PNEC}} \text { water }
$$

where $\mathrm{MC}$ is the measured concentration of each OCP in water, and $\mathrm{PNEC}_{\text {water }}$ is the predicted noeffect concentration calculated using the ECOSAR software model (Song et al. 2016). This model is mainly used to calculate acute toxicity values of organic matter. Because there were no toxicity values for loaches, the acute toxicity value of fish was used instead. Similarly, PNEC $_{\text {sediment }}$ could be deduced from the $\mathrm{PNEC}_{\text {water }}$ as determined in a previ-

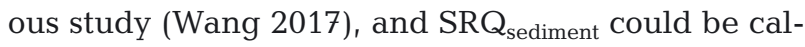
culated using Eq. (2), where PNEC $_{\text {water }}$ was used instead of $\mathrm{PNEC}_{\text {sediment. Because of the vital role of }}$ zooplankton and phytoplankton in the growth of loaches when formulated feed is not supplied, the survival of these organisms was also calculated. However, as they only occur in water, only Eq. (2) was used.

\subsection{Dietary risk of loaches before sale}

To assess the dietary risk posed by loaches collected at the end of the co-culture season (shortly before sale), the estimated daily intake (EDI) of each detected OCP residue in the loach samples was calculated as follows:

$$
\mathrm{EDI}=(C \times R) / \mathrm{BW}
$$

where $C$ is the average concentration of each OCP

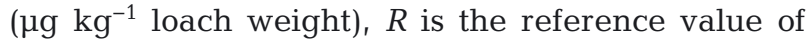
national fish consumption $\left(0.0308 \mathrm{~kg}\right.$ fish weight $\mathrm{d}^{-1}$ based on the national total diet survey) (Zhang et al. 2013), and BW is the average body weight of Chinese residents (60 kg). The EDI value was expressed as a percentage of the acceptable daily intake (ADI) value (Granados-Galván et al. 2015), with values >10\% indicating a potential risk associated with loach consumption (Song et al. 2017).

\section{RESULTS}

\subsection{Occurrence of OCPs detected in the HHRT}

The concentration of total OCPs in water and sediment uniformly decreased through time, following the order May > August > October (Fig. 2). At the beginning of the co-culture season (May), endosulfan II and heptachlor accounted for over $50 \%$ of the OCPs found in the water and sediment samples, with concentrations of $664.26 \mathrm{ng} \mathrm{l^{-1 }}$ and $5.30 \mu \mathrm{g} \mathrm{kg}^{-1}$, respectively (Table 1). In August and October, heptachlor, heptachlor epoxide, endosulfan II, DDTs,
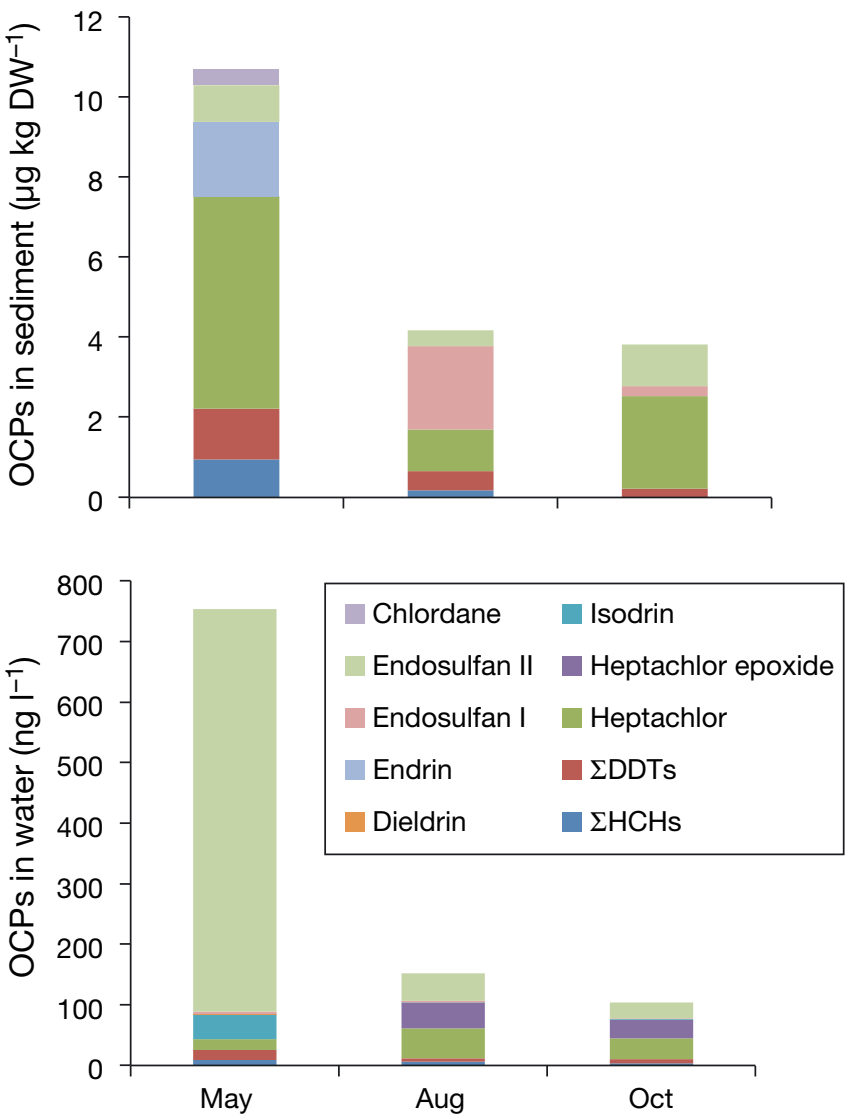

Fig. 2. Distribution and concentration of total organochlorine pesticides (OCPs) in (A) sediment and (B) water from the Honghe Hani Rice Terraces. DDT: dichlorodiphenyl trichlorothanes; HCJs: hexachlorocyclohexanes; DW: dry weight 


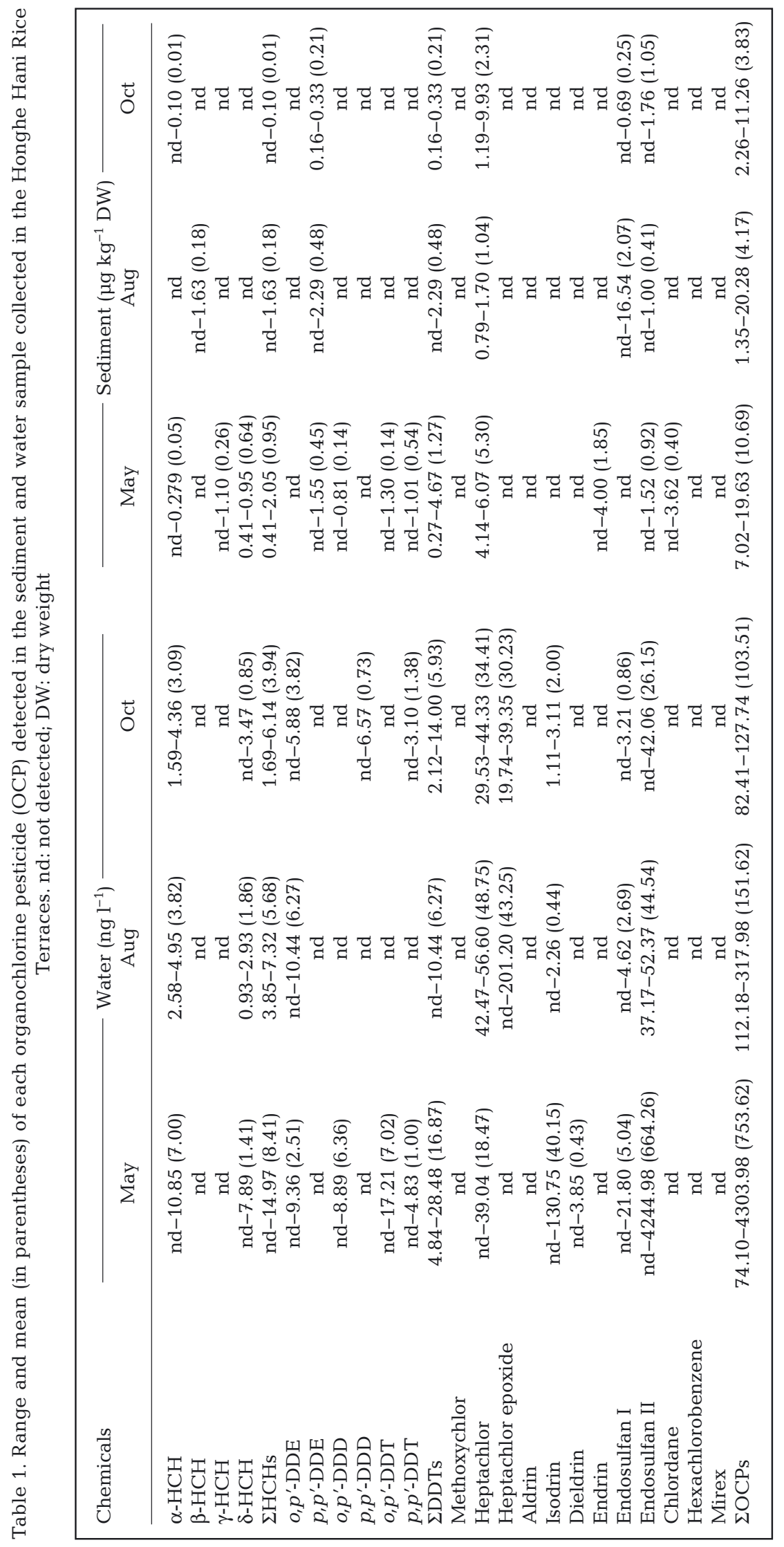

$\mathrm{HCHs}$, endosulfan I, and isodrin comprised the total OCPs (in order from high to low concentration), while endosulfan I, heptachlor, endosulfan II, DDTs, and HCHs comprised the total OCPs in the sediment (also in order from high to low). Overall, the residual profile of OCPs in the coculture environment differed over time. Specifically, sediment and water samples collected in August and October were clustered together on the nMDS plot and differed from those collected at the beginning of the season (May) (Fig. 3). For HCHs (Table 1$), \alpha$ - and $\delta$ - $\mathrm{HCH}$ were detected in the water, with $\alpha-\mathrm{HCH}$ accounting for most of the total $\mathrm{HCHs}$, while in the sediment, all isomers $(\alpha-$, $\beta-, \gamma-$, and $\delta-\mathrm{HCH})$ were detected, but $\gamma$ - and $\delta-\mathrm{HCH}$ accounted for the majority at the beginning of the coculture period and $\alpha$ - and $\beta-\mathrm{HCH}$ were rarely detected at end of the period. Evaluation of DDTs (Table 1) revealed that $o, p^{\prime}$-DDT (DDD), $o, p^{\prime}$ $\mathrm{DDE}$, and $p, p^{\prime}$-DDD accounted for the majority of DDTs in the water in May, August, and October, respectively. However, $p, p^{\prime}$-DDT and its metabolite $p, p^{\prime}$-DDE accounted for most of the DDT in the sediment in May, after which only $p, p^{\prime}$-DDE was detected. Methoxychlor, aldrin, hexachlorobenzene, and mirex were not detected in the co-culture environment during the experimental period.

\subsection{Survival risk of HHRT loaches}

Similar to other fish, loaches are affected by environmental organic pesticide residues. Through the calculation of SRQ, it was found that OCPs in the environment influenced the survival risk of fish (and by proxy of loaches), but not of plankton (Fig. 4). Most OCPs did not affect the survival risk to loaches. However, the SRQ for heptachlor was 5.29, 1.17, and 2.44 in May, August, and October, respectively, indicating that hep- 

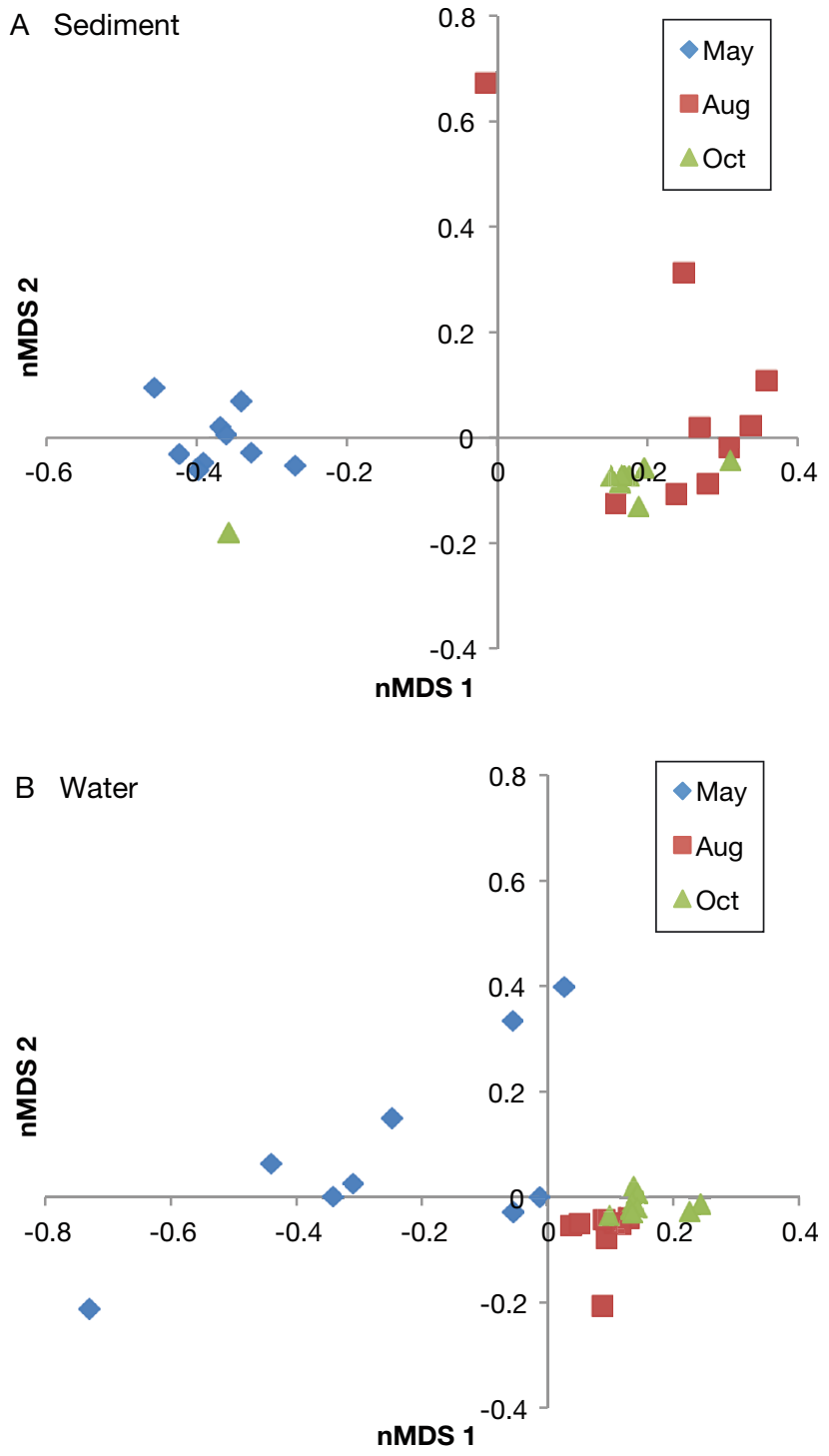

Fig. 3. Non-metric multidimensional scaling (nMDS) ordination of Bayesian distances among all (A) sediment and (B) water samples. Samples from May showed significant separation from August and October (PERMANOVA, p < 0.01) tachlor posed a survival risk to loaches throughout the entire co-culture season. In May the SRQs for endrin and chlordane were 1.66 and 1.90, respectively, indicating that these 2 OCPs posed a survival risk to loaches at the beginning of the co-culture season. The SRQs of plankton for all types of OCPs at different sampling times were all less than 1, indicating that the natural food resources of the loaches living in HHRT were not affected by OCPs.

\subsection{Assessment of risk associated with consumption of loaches}

As shown in Table 2, residual OCPs were generally not detected in the muscle of loaches sent to market. Indeed, only HCHs, DDTs, and hexachlorobenzene were detected, with residual values of $0.45,3.56$, and $0.52 \mu \mathrm{g} \mathrm{kg}^{-1}$, respectively. The EDI values of HCHs and DDTs accounted for less than $0.02 \%$ of the ADI, indicating that the 2 categories of OCPs pose little risk to human health. It should be noted that the EDI of hexachlorobenzene was not calculated because there was no ADI value available.

\section{DISCUSSION}

The present study investigated occurrences of 22 OCPs in water, sediment, and loaches in the HHRT, aiming to explore how OCPs influence the survival and edibility of loaches living in the newly introduced rice-fish co-culture system. The total OCPs ( $\Sigma$ OCPs) in all sediments collected in the HHRT of southwest China ranged from 3.83 to $10.69 \mu \mathrm{g} \mathrm{kg}^{-1}$, which is similar to the residual levels present in the rice paddy fields of northeast China (1.01 to $12.04 \mu \mathrm{g}$ $\mathrm{kg}^{-1}$ ) (Zhang et al. 2016). These results confirm that

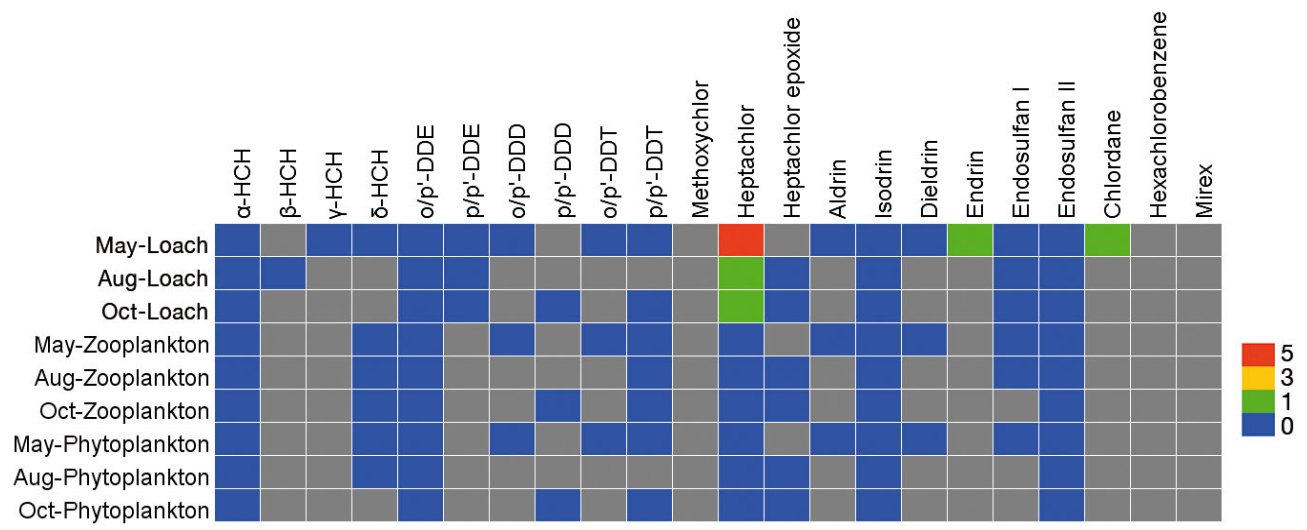

Fig. 4. Survival risk quotient (SRQ) for loaches, zooplankton and phytoplankton associated with each organochlorine pesticide. Sample collection in 2017 in Yunnan Province, China. Gray: not detected 
the levels of OCPs remaining in the paddy soils are similar between regions, even though OCPs have been banned for decades.

Some OCPs, such as heptachlor, heptachlor epoxide, endosulfan I, endosulfan II, DDTs, and HCHs, were abundant in the co-culture environment of the HHRT. Additionally, DDTs and HCHs were persistently present at lower concentrations in the sediment, with values ranging from 0.21 to 1.27 and 0.01 to $0.95 \mu \mathrm{g} \mathrm{kg}^{-1}$, respectively. The residual levels of DDTs in the sediments in this study were similar to those in paddy soils of Northeast China (mean: $1.50 \mu \mathrm{g} \mathrm{kg}^{-1}$ ) (Zhang et al. 2016) but were much lower than those in soils from the Pearl River Delta (mean: $24.7 \mu \mathrm{g} \mathrm{kg}^{-1}$ ) (Fu et al. 2003), Beijing (mean: $77.18 \mu \mathrm{g} \mathrm{kg}^{-1}$ ) (Li et al. 2008, Tao 2008), and the Yangtze River Delta (mean: $56.2 \mu \mathrm{g} \mathrm{kg}^{-1}$ ) (Sun et al. 2016). When compared with DDTs, the residual levels of $\mathrm{HCHs}$ in sediments were comparable to those in paddy soils of Northeast China (mean: $1.99 \mu \mathrm{g} \mathrm{kg}^{-1}$ ) (Zhang et al. 2016), to surface sediment from the Pearl River Delta (mean: $3.01 \mathrm{\mu g}$ $\mathrm{kg}^{-1}$ ) (Fu et al. 2003), and to agricultural sediment from the Yangtze River Delta (mean: $2.46 \mu \mathrm{g} \mathrm{kg}^{-1}$ ) (Sun et al. 2016). These regional variations in sediment concentrations of DDTs among natural systems may be a result of their proximity to agricultural systems. Conversely, the sediment concentrations of $\mathrm{HCHs}$ did not vary significantly among regions. Because of the persistence and lipid solubility of these compounds, residual levels of DDTs and $\mathrm{HCHs}$ were detected in the muscle of loaches. However, the levels were relatively low $(<0.1 \%)$, indicating that residual DDTs and HCHs in loaches being sent to market do not pose a threat to human health via consumption.

DDTs and HCHs were banned years ago, but still remain in the environment. The sources of these pesticides deserves attention. The ratios of DDD/DDE and $\mathrm{DDT} /(\mathrm{DDD}+\mathrm{DDE})$ are generally used to assess the extent of DDT decomposition because DDT is degraded into DDE and DDD under aerobic and anaerobic conditions, respectively (Hu et al. 2010, Pan et al. 2016). In the present study, higher DDT/ (DDD+DDE) ratios were found in the water (range: 0.58 to 1.81 ) and sediment (range: 0.59 to 2.04 ) at the beginning of the co-culture period, while no DDT isomers were detected in the muscle of loaches being sent to market.
These results indicate that the HHRT may have new inputs of DDTs, but that the aquatic organisms are not being affected by residual DDTs. This could have occurred because the co-culture fish species were only $1 \mathrm{yr}$ old. Generally, $\alpha-\mathrm{HCH}$ is the predominant isomer in technical $\mathrm{HCH}$, and this isomer accounts for 55 to $80 \%$ of the total HCHs (Cui et al. 2015). In this study, abundant residual levels of $\alpha-\mathrm{HCH}$ in the water were detected at values ranging from 1.59 to $10.85 \mathrm{ng} \mathrm{l}^{-1}$, while they were rarely detected in the sediment. No residual $\gamma-\mathrm{HCH}$ was detected in the water, and very little was detected in the sediment, indicating that new inputs of HCHs may exist, but they are not being deposited in the sediment. In addition, the input of these OCPs may be due to remote transportation through the atmosphere, as most OCPs have been banned (Xing et al. 2010, Sun et al. 2018). Therefore, monitoring of OCP residues needs to continue.

On the nMDS plot, samples collected during May clustered together, confirming that the highest concentration of total OCPs occurred at the beginning of the co-culture season. This may be because the surface sediment and water environment of rice fields are affected by farming. In addition, the continuous flow of water will also affect the distribution of pesticides. Endosulfan II and heptachlor were largely detected in water and sediment, respectively; however, only heptachlor posed a high potential risk to the entire food chain of cultured aquatic species. This may be because its highly stable structure enables heptachlor to persist in the environment for decades, posing an ecological risk to zooplankton and phytoplankton (Purnomo et al. 2013). Magnani et al. (1978) reported that growth, productivity, chlorophyll a content and cell size distribution of phytoplankton were inhibited by the presence of chlordane and heptachlor (Magnani et al. 1978). Overall, the results of the present study indicate that heptachlor remaining in the HHRT should receive more attention.

Table 2. Estimated daily intake (EDI) value, expressed as a percentage of the acceptable daily intake (ADI) value, used to determine the risk to human health of consuming loaches living in Honghe Hani Rice Terraces. HCHs: hexachlorocyclohexanes; DDTs: dichlorodiphenyl trichloroethanes; WW: wet weight; BW: body weight; (-) not available

\begin{tabular}{|lccccc|}
\hline Chemicals & $\begin{array}{c}\text { Mean } \\
\left(\mu \mathrm{kg}^{-1} \mathrm{WW}\right)\end{array}$ & $\mathrm{SE}$ & $\begin{array}{c}\mathrm{EDI} \\
\left(\mu \mathrm{kg}^{-1} \mathrm{BW}\right)\end{array}$ & $\begin{array}{c}\mathrm{ADI} \\
\left(\mu \mathrm{kg}^{-1} \mathrm{BW}\right)\end{array}$ & EDI/ADI \\
\hline$\sum$ HCHs & 0.45 & 0.45 & 0.0002 & 5 & $0.00 \%$ \\
$\sum$ DDTs & 3.56 & 3.46 & 0.0018 & 10 & $0.02 \%$ \\
Hexachlorobenzene & 0.52 & 0.19 & 0.0003 & - & - \\
\hline
\end{tabular}




\section{CONCLUSIONS}

The present study aimed to explore how residual OCPs influence the survival and edibility of loaches living in the newly introduced rice-fish co-culture system in the World Heritage HHRT. Among all 22 selected OCPs, heptachlor, heptachlor epoxide, endosulfan I, endosulfan II, DDTs, and HCHs were detected in abundance. Although some DDTs and $\mathrm{HCHs}$ may have been recently introduced, they were persistently present in low concentrations in water, sediment, and the muscle tissue of loaches, posing no potential survival risk for loaches or health risk for human consumption. Additionally, heptachlor accounted for most of the OCPs found at the beginning of the co-culture season and influenced the growth of zooplankton and phytoplankton, and therefore the survival of loaches; however, no residual levels were detected in the muscle of loaches. Nevertheless, heptachlor in the HHRT should receive more attention in future studies.

Acknowledgements. The authors thank the anonymous reviewers for their constructive comments. This work was supported by the Central Public-Interest Scientific Institution Basal Research Fund, CAFS (No. 2018HY-ZD0605), and the National Quality and Safety Project of the Aquatic Products of China (GJFP201700903).

\section{LITERATURE CITED}

Cui L, Ge J, Zhu Y, Yang Y, Wang J (2015) Concentrations, bioaccumulation, and human health risk assessment of organochlorine pesticides and heavy metals in edible fish from Wuhan, China. Environ Sci Pollut Res Int 22: 15866-15879

*Daley JM, Paterson G, Drouillard KG (2014) Bioamplification as a bioaccumulation mechanism for persistent organic pollutants (POPs) in wildlife. Rev Environ Contam Toxicol 227:107-155

Fu J, Mai B, Sheng G, Zhang G and others (2003) Persistent organic pollutants in environment of the Pearl River Delta, China: an overview. Chemosphere 52:1411-1422

Godfray HCJ, Beddington JR, Crute IR, Haddad L and others (2010) Food security: the challenge of feeding 9 billion people. Science 327:812-818

Granados-Galván IA, Rodríguez-Meza DG, Luna-González A, González-Ocampo HA (2015) Human health risk assessment of pesticide residues in snappers (Lutjanus) fish from the Navachiste Lagoon complex, Mexico. Mar Pollut Bull 97:178-187

Gu H, Jiao Y, Liang L (2012) Strengthening the socioecological resilience of forest-dependent communities: the case of the Hani Rice Terraces in Yunnan, China. For Policy Econ 22:53-59

Hu G, Dai J, Mai B, Luo X and others (2010) Concentrations and accumulation features of organochlorine pesticides in the Baiyangdian Lake freshwater food web of North China. Arch Environ Contam Toxicol 58:700-710
Kim JH, Smith A (2001) Distribution of organochlorine pesticides in soils from South Korea. Chemosphere 43: 137-140

Lal R, Pandey G, Sharma P, Kumari K and others (2010) Biochemistry of microbial degradation of hexachlorocyclohexane and prospects for bioremediation. Microbiol Mol Biol Rev 74:58-80

ㄴi X, Gan Y, Yang X, Zhou J, Dai J, Xu M (2008) Human health risk of organochlorine pesticides (OCPs) and polychlorinated biphenyls (PCBs) in edible fish from Huairou Reservoir and Gaobeidian Lake in Beijing, China. Food Chem 109:348-354

Mäder P, Fließach, Dubois D, Gunst L, Fried P, Niggli U (2002) Soil fertility and biodiversity in organic farming. Science 296:1694-1697

* Magnani B, Powers CD, Wurster CF, O'Connors HB Jr (1978) Effects of chlordane and heptachlor on the marine dinoflagellate, Exuviella baltica, Lohmann. Bull Environ Contam Toxicol 20:1-8

* Mahmoud AF, Ikenaka Y, Yohannes YB, Darwish WS and others (2016) Distribution and health risk assessment of organochlorine pesticides (OCPs) residue in edible cattle tissues from northeastern part of Egypt: high accumulation level of OCPs in tongue. Chemosphere 144:1365-1371

* Mrema EJ, Rubino FM, Brambilla G, Moretto A, Tsatsakis AM, Colosio C (2013) Persistent organochlorinated pesticides and mechanisms of their toxicity. Toxicology 307 : $74-88$

Oksanen J, Blanchet GF, Kindt R, Legendre P and others (2006) vegan: community ecology package. R package version 2.0-4

*Pan H, Geng J, Qin Y, Tou F, Zhou J, Liu M, Yang Y (2016) PCBs and OCPs in fish along coastal fisheries in China: distribution and health risk assessment. Mar Pollut Bull 111:483-487

* Purnomo AS, Mori T, Putra SR, Kondo R (2013) Biotransformation of heptachlor and heptachlor epoxide by white-rot fungus Pleurotus ostreatus. Int Biodeterior Biodegradation 82:40-44

Q Qiu X, Zhu T, Yao B, Hu J, Hu S (2005) Contribution of dicofol to the current DDT pollution in China. Environ Sci Technol 39:4385-4390

Singh RK (2007) Indigenous agricultural knowledge in rainfed rice based farming systems for sustainable agriculture: learning from Indian farmers. In: Boon EK, Hens L (eds) Indigenous knowledge systems and sustainable development: relevance for Africa. Kamal-Raj Enterprises, New Deli, p 101-110

* Singh Z (2016) Toxic effects of organochlorine pesticides: a review. Am J BioSci 4:11-18

* Song C, Zhang C, Fan L, Qiu L and others (2016) Occurrence of antibiotics and their impacts to primary productivity in fishponds around Tai Lake, China. Chemosphere 161:127-135

* Song C, Li L, Zhang C, Kamira B and others (2017) Occurrence and human dietary assessment of sulfonamide antibiotics in cultured fish around Tai Lake, China. Environ Sci Pollut Res Int 24:17493-17499

* Sun J, Pan L, Zhan Y, Lu H and others (2016) Contamination of phthalate esters, organochlorine pesticides and polybrominated diphenyl ethers in agricultural soils from the Yangtze River Delta of China. Sci Total Environ 544: 670-676

Sun Y, Yuan GL, Li J, Tang J, Wang GH (2018) Highresolution sedimentary records of some organochlorine 
pesticides in Yamzho Yumco Lake of the Tibetan Plateau: concentration and composition. Sci Total Environ 615:469-475

Tao S (2008) Organochlorine pesticides contaminated surface soil as reemission source in the Haihe Plain, China. Environ Sci Technol 42:8395-8400

Wang J (2017) Ecological risk assessment of organochlorine pesticides in Daling River basin. MSc thesis, Dalian Maritime University (in Chinese)

Xie J, Hu L, Tang J, Wu X and others (2011) Ecological mechanisms underlying the sustainability of the agricultural heritage rice-fish coculture system. Proc Natl Acad Sci USA 108:E1381-E1387

Xing XL, Qi SH, Zhang Y, Yang D, Odhiambo JO (2010) Organochlorine pesticides (OCPs) in soils along the eastern slope of the Tibetan Plateau. Pedosphere 20: 607-615

Zhang H, Zhao X, Ni Y, Lu X and others (2010) PCDD/Fs and PCBs in sediments of the Liaohe River, China: levels,

Editorial responsibility: Louis Lebel,

Chiang Mai, Thailand distribution, and possible sources. Chemosphere 79: 754-762

Khang L, Li J, Zhao Y, Li X, Wen S, Shen H, Wu Y (2013) Polybrominated diphenyl ethers (PBDEs) and indicator polychlorinated biphenyls (PCBs) in foods from China: levels, dietary intake, and risk assessment. J Agric Food Chem 61:6544-6551

Z Zhang H, Lu X, Zhang Y, Ma X, Wang S, Ni Y, Chen J (2016) Bioaccumulation of organochlorine pesticides and polychlorinated biphenyls by loaches living in rice paddy fields of Northeast China. Environ Pollut 216:893-901

Zhang Y, Min Q, Zhang C, He L and others (2017) Traditional culture as an important power for maintaining agricultural landscapes in cultural heritage sites: a case study of the Hani terraces. J Cult Herit 25:170-179

* Zhao Z, Zhang L, Wu J, Fan C (2013) Residual levels, tissue distribution and risk assessment of organochlorine pesticides (OCPs) in edible fishes from Taihu Lake, China. Environ Monit Assess 185:9265-9277

Submitted: August 29, 2018; Accepted: March 11, 2019 Proofs received from author(s): May 10, 2019 